\title{
DYNAMIC ANALYSIS OF LARGE STRAIN DEFORMATION OF FLEXIBLE PIPES CONVEYING TWO-PHASE FLUIDS PART I: LINEAR VIBRATION ANALYSIS.
}

\author{
A. T. Adebusoye ${ }^{1}$, T. A. Fashanu ${ }^{2, *}$ and A. A. Oyediran ${ }^{3}$ \\ 1, 3, Department of MeChanical Engineering, University of Lagos, AKoKA, LAGos State, NIGERIA \\ 2, DEPARTMENT OF SYSTEMS ENGINEERING, UNIVERSITY OF LAGOS, AKOKA, LAGOS STATE, NIGERIA \\ E-mail addresses: ${ }^{1}$ abeti1111@gmail.com, 2 tfashanu@unilag.edu.ng, ${ }^{3}$ ayooyediran@unilag.edu.ng
}

\begin{abstract}
This work presents dynamic response of large strain deformed subsea flowlines and jumpers conveying two-phase fluid. Large strain deformation theory is used to analyze the effects of high pressure-high temperature two-phase flow on critical velocity in flexible pipes. Starting with natural frequency of simply supported flexible pipes, corresponding nonlinear transport equations are derived and subsequently expanded using the method of multiple scales perturbation. Frequency response obtained from the linearized leading order equations established that buckling and flutter-like instabilities attend critical velocities. Furthermore, the buckling velocity of transverse pipe decreases with increasing temperature, pressure or tension. Clearly, the transverse buckling velocity is higher in large strain deformation model compared with small strain model. In addition; whereas the critical velocity in the longitudinal direction is independent of temperature, pressure and tension for small strain models; these variables determine the path to failure in large strain deformed pipes.
\end{abstract}

Keywords: Large strain deformation, small strain deformation, simply supported pipe.

\section{INTRODUCTION}

Flow lines and jumpers belong to a class of flexible pipes that are widely deployed for offshore deep water exploration and production [1]. Often, these flexible pipes undergo large strain deformations than small strain deformations.

Stability of pipes conveying fluid is a subject of utmost concern to research and development scientists as well as field design engineers. Most existing works modelled deformation resulting from fluid/solid pipe interaction with small strain theory. For example, [2] used the small strain deformation theory to report that a simply supported straight pipe conveying fluid would lose stability first by buckling. Later, it will undergo a coupled-mode flutter. Similarly, Lee and Chung [3] modeled a straight pipe with large strains conveying single phase fluid and obtained the natural frequencies and responses of the pipe. However, some interesting physics due to the large strain deformation were omitted because the effects of temperature, tension and pressure were neglected.

This work derived the model of a large strain deformed straight pipe conveying two-phase flow, under the effects of thermal strains, pressurization and tension. Adopting the direct method of multiple time scale perturbation, the nonlinear equations were expanded. Subsequently, the linear natural frequencies were obtained numerically. This provided a basis for the analysis of the effects of large strain deformation compared to small strain deformation on transverse and longitudinal critical velocities of fluid flow in the pipe.

\section{THEORECTICAL BACKGROUND}

Consider a pipe conveying two-phase fluids that is deformed with large strains. Adopting linear stresses and nonlinear strains as introduced by [3] for a 
straight pipe with the thermal strains, pressurization and tension. The strains of the pipe due to the elastic large deformation is given by:

$$
\begin{gathered}
\varepsilon_{x}^{N L}=\bar{u}^{\prime}+\frac{1}{2}\left(\bar{u}^{\prime 2}+\bar{w}^{\prime 2}\right)-y\left(1+\bar{u}^{\prime}\right) \bar{w}^{\prime \prime} \\
+\frac{1}{2} y^{2} \bar{w}^{\prime 2}-\alpha \bar{\theta}+\frac{\bar{T}_{0}-\bar{P}}{E A} \\
\varepsilon_{x}^{L}=\bar{u}^{\prime}-y \bar{w}^{\prime \prime}-\alpha \bar{\theta}+\frac{\bar{T}_{0}-\bar{P}}{E A}
\end{gathered}
$$

Where $\bar{u}$ and $\bar{w}$ are the longitudinal and transverse displacements of the pipe, $\bar{\theta}$ is the temperature change of the pipe conveying fluid, $\alpha$ is coefficient of thermal expansion, $\bar{T}_{0}$ is tension in the pipe, $\bar{P}$ is pressurization. $\varepsilon_{x}^{N L}$ is the nonlinear strain of the pipe and $\varepsilon_{x}^{L}$ is the linearized strain of the pipe. However, the pipe is assumed not to have yielded when subjected to linear stress given by:

$$
\sigma_{x}^{L}=E \varepsilon_{x}^{L}
$$

Thus, the strain energy of the pipe conveying fluid due to large elastic deformation is given by:

$$
\mathcal{V}=\frac{1}{2} \int \sigma_{x}^{L} \varepsilon_{x}^{N L} d V
$$

For two phase flow, the kinetic energy is the sum of the kinetic energy of the pipe and the kinetic energies of the two fluids. It is given by:

$$
\begin{aligned}
T=\frac{1}{2} m_{p} \int\left(\dot{\bar{u}}^{2}\right. & \left.+\dot{\bar{w}}^{2}\right) d x \\
& +\frac{1}{2} m_{f_{1}} \int\left(\left(\dot{\bar{u}}+\bar{v}_{f 1}\left(1+\bar{u}^{\prime}\right)\right)^{2}\right. \\
& \left.+\left(\dot{\bar{w}}+\bar{v}_{f 1} \bar{w}^{\prime}\right)^{2}\right) d x \\
& +\frac{1}{2} m_{f_{2}} \int\left(\left(\dot{\bar{u}}+\bar{v}_{f 2}\left(1+\bar{u}^{\prime}\right)\right)^{2}\right. \\
& \left.+\left(\dot{\bar{w}}+\bar{v}_{f 2} \bar{w}^{\prime}\right)^{2}\right) d x
\end{aligned}
$$

Using the energy method, the Lagrangian $\mathcal{L}$, of the system is given by:

$$
\mathcal{L}=\mathcal{T}-\mathcal{V}
$$

Applying the Hamiltonian principle to minimize the Lagrangian of the system as stated in equation (6):

$$
\delta \int_{t 1}^{t 2} \mathcal{L} d t+\int_{t 1}^{t 2} \delta \mathcal{W} d t=0
$$

Where $\mathcal{T}$ and $\mathcal{V}$ are the total kinetic and potential energies of the pipe. The $\delta \mathcal{W}$ is the virtual work due to non-conservative forces imposed on the pipe, [4]. Substituting Equations (1) - (6) into Equation (7), the nonlinear vibration in the longitudinal direction could be derived as (u-equation):

$$
\begin{aligned}
\left(m_{p}+m_{f 1}+m_{f 2}\right) \ddot{\bar{u}}+2\left(m_{f 1} \bar{v}_{f 1}+m_{f 2} \bar{v}_{f 2}\right) \dot{\bar{u}}^{\prime}+\left(m_{f 1} \bar{v}_{f 1}^{2}+m_{f 2} \bar{v}_{f 2}^{2}\right) \bar{u}^{\prime \prime} \\
+E A\left(-\bar{u}^{\prime \prime}+\frac{3 \bar{u}^{\prime} \bar{u}^{\prime \prime}}{4}+\frac{3 \bar{w}^{\prime} \bar{w}^{\prime \prime}}{2}\right)-E I\left(\frac{3 \bar{w}^{\prime \prime} \bar{w}^{\prime \prime \prime}}{2}\right)-E A^{\prime} \bar{u}^{\prime}+\frac{3 E A^{\prime} \bar{u}^{\prime 2}}{4}+\frac{E A^{\prime} \bar{w}^{\prime 2}}{2} \\
+\frac{\boldsymbol{E} \propto}{\mathbf{2}}\left(A \bar{\theta}^{\prime} \bar{u}^{\prime}+\bar{\theta} A^{\prime} \bar{u}^{\prime}+A \bar{\theta} \bar{u}^{\prime \prime}\right)+\frac{\boldsymbol{E} \propto}{\mathbf{2}}\left(\frac{A \bar{\theta}^{\prime}}{2}+\frac{A^{\prime} \bar{\theta}}{2}\right) \\
+\left(\frac{(\bar{P} A)^{\prime}}{2}-\left(\frac{\bar{T}_{0}-\bar{P} A}{2 A}\right) \bar{u}^{\prime \prime}-\frac{(\bar{P} A)^{\prime}}{2} \bar{u}^{\prime}\right)=0
\end{aligned}
$$

In addition, the nonlinear vibration in the transverse direction could be described by ( $w$-equation):

$$
\begin{aligned}
\left(m_{p}+m_{f 1}+m_{f 2}\right) & \ddot{\bar{w}}+2\left(m_{f 1} \bar{v}_{f 1}+m_{f 2} \bar{v}_{f 2}\right) \dot{\bar{w}}^{\prime}+\left(m_{f 1} \bar{v}_{f 1}^{2}+m_{f 2} \bar{v}_{f 2}^{2}\right) \bar{w}^{\prime \prime} \\
& +E I\left(\bar{w}^{I V}+3 u^{\prime \prime} \bar{w}^{\prime \prime \prime}+\frac{3}{2} \bar{u}^{\prime \prime \prime} \bar{w}^{\prime \prime}+\frac{3}{2} \bar{u}^{\prime} \bar{w}^{I V}\right)-E A\left(\frac{1}{2} \bar{w}^{\prime} \bar{u}^{\prime \prime}+\frac{1}{2} \bar{w}^{\prime \prime} \bar{u}^{\prime}\right) \\
& +\frac{E \propto}{2}\left(A \bar{\theta}^{\prime} \bar{w}^{\prime}+\bar{\theta} A^{\prime} \bar{w}^{\prime}+A \bar{\theta} \bar{w}^{\prime \prime}\right)+\left(\frac{(\bar{P} A)^{\prime}}{2}-\left(\frac{\bar{T}_{0}-\bar{P} A}{2 A}\right) \bar{w}^{\prime \prime}-\frac{(\bar{P} A)^{\prime}}{2} \bar{w}^{\prime}\right) \\
& +E\left(A^{\prime} \bar{w}^{\prime} \bar{u}^{\prime}+A \bar{u}^{\prime \prime} \bar{w}^{\prime}+A \bar{u}^{\prime} \bar{w}^{\prime}\right)-I\left(\bar{P}^{\prime \prime} \bar{w}^{\prime \prime}+2 \bar{P}^{\prime} \bar{w}^{\prime \prime \prime}-\left(\frac{\bar{T}_{0}}{A}-\bar{P}\right)\right) \bar{w}^{I V} \\
& +\frac{E I \propto}{2}\left(\bar{\theta}^{\prime} \bar{w}^{\prime \prime}+\bar{\theta}^{\prime} \bar{w}^{\prime \prime \prime}+\bar{\theta} \bar{w}^{I V}\right)=0
\end{aligned}
$$

Hence, the nonlinear coupled equations for the transverse and longitudinal vibrations in the dimensionless forms are as presented in Equations (10a) and (10b): 
Dynamic Analysis of LaRge Strain Deformation of FleXible PIPes Conveying Two-PhaSe FluidS,....., A. T. Adebusoye, et. al

$$
\begin{aligned}
\ddot{w}+2 & +\left(v_{f 1} \sqrt{\beta_{f 1}} \sqrt{\beta_{1}}+v_{f 2} \sqrt{\beta_{f 2}} \sqrt{\beta_{2}}\right) \dot{w}^{\prime}+\left(\beta_{f 1} v_{f 1}{ }^{2}+\beta_{f 2} v_{f 2}{ }^{2}\right) w^{\prime \prime}+\left(\dot{v}_{f 1} \sqrt{\beta_{f 1}} \sqrt{\beta_{1}}\right. \\
& \left.+\dot{v}_{f 2} \sqrt{\beta_{f 2}} \sqrt{\beta_{2}}\right) w^{\prime}+w^{I V}+\frac{3}{2}\left(u^{\prime \prime \prime} w^{\prime \prime}+2 u^{\prime \prime} w^{\prime \prime \prime}+u^{\prime} w^{I V}\right)-\frac{\eta}{2}\left(u^{\prime \prime} w^{\prime}+u^{\prime} w^{\prime \prime}\right)-\frac{\eta \delta u^{\prime} w^{\prime}}{2} \\
& +\frac{\eta}{2}\left(\delta \theta t h w^{\prime}+\theta t h^{\prime} w^{\prime}+\theta t h w^{\prime \prime}\right)+\frac{1}{\eta}\left(\frac{\theta t h^{\prime \prime} w^{\prime \prime}}{2}-\theta t h^{\prime} w^{\prime \prime \prime}-\theta t h w^{I V}\right)-T e w^{\prime \prime} \\
& +\frac{2}{\eta} \operatorname{Te} w^{I V}+\left(\operatorname{Pr}^{\prime} w^{\prime}+\delta P r w^{\prime}+\operatorname{Pr} w^{\prime \prime}\right)-\frac{1}{\eta}\left(P r^{\prime \prime} w^{\prime \prime}+2 P r^{\prime} w^{\prime \prime \prime}+\operatorname{Pr} w^{I V}\right)=0 \\
& \ddot{u}+\left(\dot{v}_{f 1} \sqrt{\beta_{f 1}} \sqrt{\beta_{1}}+\dot{v}_{f 2} \sqrt{\beta_{f 2}} \sqrt{\beta_{2}}\right)+\left(\dot{v}_{f 1} \sqrt{\beta_{f 1}} \sqrt{\beta_{1}}+\dot{v}_{f 2} \sqrt{\beta_{f 2}} \sqrt{\beta_{2}}\right) u^{\prime} \\
& +2\left(v_{f 1} \sqrt{\beta_{f 1}} \sqrt{\beta_{1}}+v_{f 2} \sqrt{\beta_{f 2}} \sqrt{\beta_{2}}\right) \dot{u}^{\prime}+\left(\beta_{f 1} v_{f 1}^{2}+\beta_{f 2} v_{f 2}^{2}\right) u^{\prime \prime}-\frac{3}{2} w^{\prime \prime} w^{\prime \prime \prime} \\
& -\eta\left(\delta u^{\prime}+\eta u^{\prime \prime}\right)-\frac{3}{4} \eta\left(\delta u^{\prime 2}+2 u^{\prime} u^{\prime \prime}\right)-\frac{\eta}{4}\left(2 w^{\prime} w^{\prime \prime}+\delta w^{\prime 2}\right)+\frac{1}{2}\left(\delta \theta t h+\theta t h^{\prime}\right) \\
& +\frac{1}{2}\left(\delta \theta t h u^{\prime}+\theta t h^{\prime} u^{\prime}+\theta t h u^{\prime \prime}\right)+\left(-\eta T e u^{\prime \prime}\right)+\left(\frac{1}{2} \operatorname{Pr}^{\prime}+\frac{1}{2} \delta . \operatorname{Pr}\right) \\
& +\left(\operatorname{Pr} u^{\prime}+\delta \operatorname{Pr} u^{\prime}+\operatorname{Pr} u^{\prime \prime}\right)=0
\end{aligned}
$$

The boundary conditions representing the physics of the problem are expressed in Equation (11):

$$
\bar{w}(0)=\frac{d^{2} \bar{w}}{d x^{2}}(0)=\bar{w}(L)=\frac{d^{2} \bar{w}}{d x^{2}}(L)=\bar{u}(0)=\bar{u}(L)=0
$$

To arrive at Equations (10a), (10b) and (11), the following dimensionless parameters were derived:

$$
\begin{gathered}
u=\frac{\bar{u}}{L}, \quad w=\frac{\bar{w}}{L}, t=\frac{\bar{t}}{T_{c}}, v_{f j}=\left(\frac{M_{j}}{E I}\right)^{1 / 2} \bar{v}_{f j} L, \quad \operatorname{Pr}=\frac{\bar{P}}{P_{c}}, \theta t h=\frac{\bar{\theta}}{\theta_{c}}, \\
\beta_{j}=\frac{m_{f j}}{\sum m_{f j}+m_{p}}, \quad \beta_{f j}=\frac{m_{f j}}{\sum m_{f j}}, T=l^{2} \sqrt{\frac{m_{p}+m_{f_{1}}+m_{f_{2}}}{E I}}, M_{j}=\sum m_{f j}+m_{p}, \theta_{c}=\frac{E I}{E A l^{2} \alpha^{\prime}} \quad P_{c}=\frac{E I}{l^{2}}, \\
T_{c}=\frac{E I}{L^{2}}, \quad v_{c}=\frac{1}{L} \sqrt{\frac{E I}{m_{f_{1}+m_{f_{2}}}}}, \delta=\frac{A^{\prime} L}{A}, \quad \eta=\frac{E A L^{2}}{E I}
\end{gathered}
$$

Initial tension: $T e=\frac{\bar{T}_{0} L^{2}}{E I}$, Flexibility, $\eta=\frac{E A L^{2}}{E I}$, Initial Presure: $\operatorname{Pr}=\frac{\bar{P} L^{2}}{E I}$,

In these equations, $u$ and $w$ are the dimensionless displacements in the longitudinal and transverse directions respectively, $\left(v_{f j}\right)$ is the flow velocities of the constituent phases/components used in the analysis of the dynamics of the system, $\left(\beta_{j}\right)$ is the mass ratio which relates the mass of a fluid phase to the total mass of the fluids and the pipe as derived by [4] for a single phase fluid, ( $\beta_{f j}$ ) is the mass ratio which relates the mass of a fluid phase to the total fluid mass, and $\eta$ index the pipe flexibility, $T e$ is the dimensionless Tension, while $P r$ is dimensionless pressurization.

For the two phase flow the Chisolm empirical relations [5] were adopted as follows;

Voild fraction $a_{g}$

$$
\alpha_{g}=\left[1-\left(1-x\left(1-\frac{\rho_{l}}{\rho_{g}}\right)\right)^{\frac{1}{2}} \frac{1-x}{x} \frac{\rho_{g}}{\rho_{l}} \frac{v_{1}}{v_{2}}\right]^{-1}
$$

$$
\begin{aligned}
& \text { Slip Ratio, } S=\frac{v_{1}}{v_{2}}=\sqrt{1-x\left(1-\frac{\rho_{l}}{\rho_{g}}\right)} \\
& \text { Mixture velocity, } V_{m}=\alpha_{g} v_{1}+(13 \mathrm{~b})
\end{aligned}
$$

Where $\rho_{l}$ and $\rho_{g}$ are the densities of the liquid and gaseous phases respectively, $x$ is the mixture quality. $v_{1}$ and $v_{2}$ are the superficial flow velocities of the gaseous and liquid phases respectively.

\section{METHOD OF SOLUTION}

Approximate solutions are obtained with direct multiple scale perturbation technique in the form:

$$
\begin{aligned}
w\left(x, T_{0}, T_{1}, T_{2}\right)= & \epsilon w_{1}\left(x, T_{0}, T_{1}, T_{2}\right)+\epsilon^{2} w_{2}\left(x, T_{0}, T_{1}, T_{2}\right) \\
& +\epsilon^{3} w_{3}\left(x, T_{0}, T_{1}, T_{2}\right) ; \quad(14 \mathrm{a}) \\
u\left(x, T_{0}, T_{1}, T_{2}\right)= & \epsilon u_{1}\left(x, T_{0}, T_{1}, T_{2}\right)+\epsilon^{2} u_{2}\left(x, T_{0}, T_{1}, T_{2}\right) \\
& +\epsilon^{3} u_{3}\left(x, T_{0}, T_{1}, T_{2}\right)
\end{aligned}
$$

The time derivatives operators can be written as:

$$
\left.\begin{array}{c}
\frac{d}{d t}=D_{0}+\epsilon D_{1}+\cdots \\
\frac{d^{2}}{d t^{2}}=D_{0}^{2}+2 \epsilon D_{0} D_{1}+\cdots
\end{array}\right\}
$$


Performing the derivative operation and equating the coefficients of $\epsilon^{i}, i=1,2,3$. successively to zero gave;

$$
\begin{aligned}
& \epsilon^{1}: D_{0}^{2} w_{1}+2 \sqrt{\beta}\left(v_{\mathrm{f} 1} \beta_{\mathrm{f} 1}+v_{\mathrm{f} 2} \beta_{\mathrm{f} 2}\right) D_{0} w_{1}{ }^{\prime}+\left(P r-T e+v_{\mathrm{f} 1}^{2} \beta_{\mathrm{f} 1}+v_{\mathrm{f} 2}^{2} \beta_{\mathrm{f} 2}+\frac{\theta \text { th }}{2}\right) w_{1}{ }^{\prime \prime}+w_{1}^{\mathrm{iv}} \\
&-(P r+2 T e-\theta t h) \frac{w_{1}^{\mathrm{iv}}}{\eta}=0 \\
& \epsilon^{1}: \quad D_{0}^{2} u_{1}+2 \sqrt{\beta}\left(v_{\mathrm{f} 1} \beta_{\mathrm{f} 1}+v_{\mathrm{f} 2} \beta_{\mathrm{f} 2}\right) D_{0} u_{1}{ }^{\prime}+\left(P r-T e+v_{\mathrm{f} 1}^{2} \beta_{\mathrm{f} 1}+v_{\mathrm{f} 2}^{2} \beta_{\mathrm{f} 2}+\frac{\theta t h}{2}-\eta\right) u_{1}{ }^{\prime \prime}=0 \\
& \epsilon^{2}: \quad D_{0}^{2} w_{2}+2 \sqrt{\beta}\left(v_{\mathrm{f} 1} \beta_{\mathrm{f} 1}+v_{\mathrm{f} 2} \beta_{\mathrm{f} 2}\right) D_{0} w_{2}{ }^{\prime}+\left(P r-T e+v_{\mathrm{f} 1}^{2} \beta_{\mathrm{f} 1}+v_{\mathrm{f} 2}^{2} \beta_{\mathrm{f} 2}+\frac{\theta \mathrm{th}}{2}\right) w_{2}{ }^{\prime \prime}+w_{1}^{\mathrm{iv}} \\
&+(P r+2 T e-\theta t h) \frac{w_{1}^{\mathrm{iv}}}{\eta} \\
&=-2 D_{0} D_{1} w_{1}-\frac{3}{2} w_{1}^{\mathrm{iv}} u_{1}{ }^{\prime}-2 \sqrt{\beta}\left(v_{\mathrm{f} 1} \beta_{\mathrm{f} 1}+v_{\mathrm{f} 2} \beta_{\mathrm{f} 2}\right) D_{0} w_{1}{ }^{\prime}+\frac{1}{2} \eta w_{1}{ }^{\prime} u_{1}{ }^{\prime \prime}-\frac{3}{2} u_{1}^{\prime \prime \prime} w_{1}{ }^{\prime \prime} \\
&+\frac{1}{2} \eta u_{1}{ }^{\prime} w_{1}{ }^{\prime \prime}+3 u_{1}{ }^{\prime \prime} w_{1}{ }^{\prime \prime}
\end{aligned}
$$

\subsection{Linear Vibration Analysis}

The leading order problem is a linear problem and a solution of the leading order problem gives the natural frequencies and mode shapes of the system. The homogenous solution of the leading order equations (15a) and (15b) can be expressed as:

$$
\begin{gathered}
w_{1}=\mathrm{e}^{\mathrm{i} T_{0} \omega_{n}} A_{n}\left(T_{1}\right) \phi_{n}(x) \\
+\mathrm{e}^{-\mathrm{i} T_{0} \omega_{n}} \phi_{n}(x) A_{n}\left(T_{1}\right) \\
u_{1}=\mathrm{e}^{\mathrm{i} T_{0} \alpha_{n}} B_{n}\left(T_{1}\right) \psi_{n}(x) \\
+\mathrm{e}^{-\mathrm{i} T_{0} \alpha_{n}} \psi_{n}(x) B_{n}\left(T_{1}\right)
\end{gathered}
$$

Where $\phi_{n}(x)$ and $\psi_{n}(x)$ are the eigenfunctions of the linear vibration of a simply supported pipe conveying fluid, [1]. $\omega_{n}$ and $\alpha_{n}$ are the transverse vibration and longitudinal vibration natural frequencies respectively. And assuming trial solutions of the form:

$$
\begin{gathered}
\phi_{n}(x)=\operatorname{Re}\left[H_{w} \cdot e^{i k_{w} \mathrm{x}}\right] \\
\psi_{n}(x)=\operatorname{Re}\left[H_{u} \cdot e^{i k_{u} \mathrm{x}}\right]
\end{gathered}
$$

Where $k_{w}$ and $k_{u}$ are wave constants in the transverse and longitudinal directions respectively and $H_{w}$ and $H_{u}$ are constants.

\subsection{Transverse and Longitudinal natural frequencies:}

Substituting the homogenous solutions (16b) and (17b) into Equation (15b), the dispersion relation for the longitudinal vibration is obtained as:

$$
\begin{aligned}
(\eta-T e+P r+ & \left.\theta t h+\beta_{f 1} v_{f 1}^{2}+\beta_{f 2} v_{f 2}^{2}\right) k_{u}^{2} \\
& -2\left(\sqrt{\beta_{1} \beta_{f 1}} v_{1}\right. \\
& \left.+\sqrt{\beta_{2} \beta_{f 2}} v_{2}\right) \alpha k_{u}+\alpha^{2} \\
& =0
\end{aligned}
$$


Substituting the homogenous solutions (16a) and (17a) into Equation (15a); the dispersion relation for the transverse vibration is obtained as:

$$
\begin{aligned}
(2 T e-P r-\theta t h) & \frac{k_{w}^{4}}{\eta} \\
& -\left(P r+\theta t h-T e+\beta_{f 1} v_{f 1}^{2}\right. \\
& \left.+\beta_{f 2} v_{f 2}^{2}\right) k_{w}^{2} \\
& -2\left(\sqrt{\beta_{1} \beta_{f 1}} v_{1}+\sqrt{\beta_{2} \beta_{f 2}} v_{2}\right) \omega k_{w} \\
& -\omega^{2}=0
\end{aligned}
$$

Following the procedure of [2] for a simply supported pipe conveying fluid, the transverse and the longitudinal natural frequencies were obtained numerically.

\section{RESULTS}

The linear vibration results are obtained by analyzing the leading order problems of the three time scale expansion.

\subsection{Validation of Model:}

The linear vibration of a pipe conveying single phase fluid but undergoing small strain deformation was elucidated by [6], as shown in Figure 1a. They reported that divergence in the first mode occurred at a dimensionless velocity of 3.146 and subsequently at a dimensionless velocity of 6.2833 . The same pattern is observed for a pipe conveying fluid and undergoing large strain deformation when the effects of temperature, pressure and tension are negligible.

For a pipe conveying two-phase fluid the variation of the longitudinal frequencies with flow velocity is shown in Figure 1c. It is notable that when the effects of temperature, pressure and tension are negligible, the same behavior is observed when a pipe conveying fluid undergoes large strain deformation and also when it undergoes small strain deformation. Similar results were obtained in the work of [7], however, they were obtained for cantilevered pipes.

\subsection{Effects of Temperature on Transverse and Axial Vibration:}

The effects of temperature variation on the large strain deformation model against the small strain deformation model for the transverse and longitudinal vibrations are shown in Figures $2 a$ and $2 b$ respectively. These results were obtained for $a$ pipe conveying two phase flows at a void fraction of 0.3 and mixture quality of 0.00037088 . . Clearly, the critical velocities of the pipe reduce with increasing temperature. This is similar to the results of [8] for a cantilevered pipe conveying single phase flow modeled with small strain deformation.

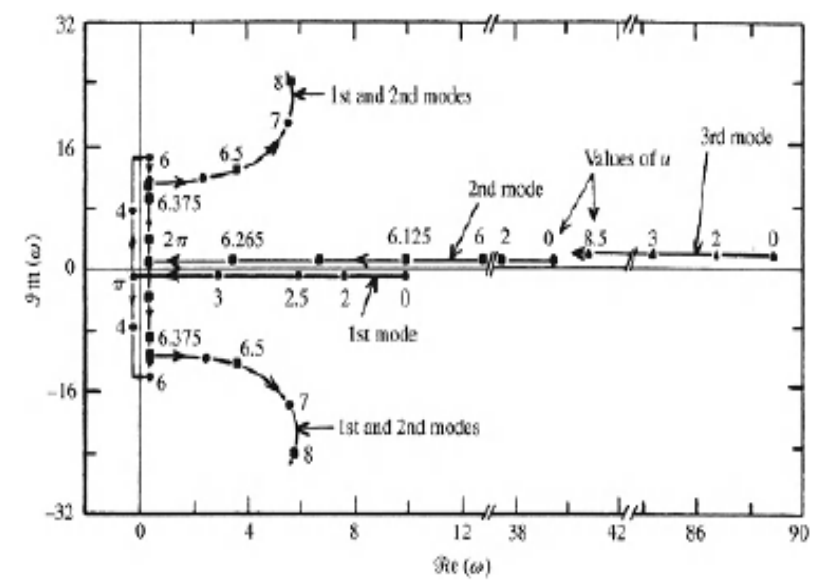

Figure 1a: Transverse Natural Frequencies for small strain deformation [6]

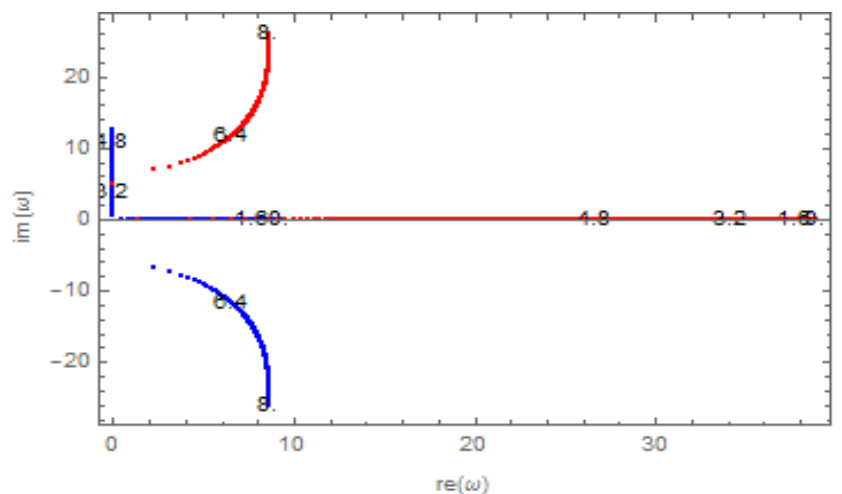

Figure 1b: Transverse Natural Frequencies for large strain deformation (neglecting the effect of temperature, pressure and tension)

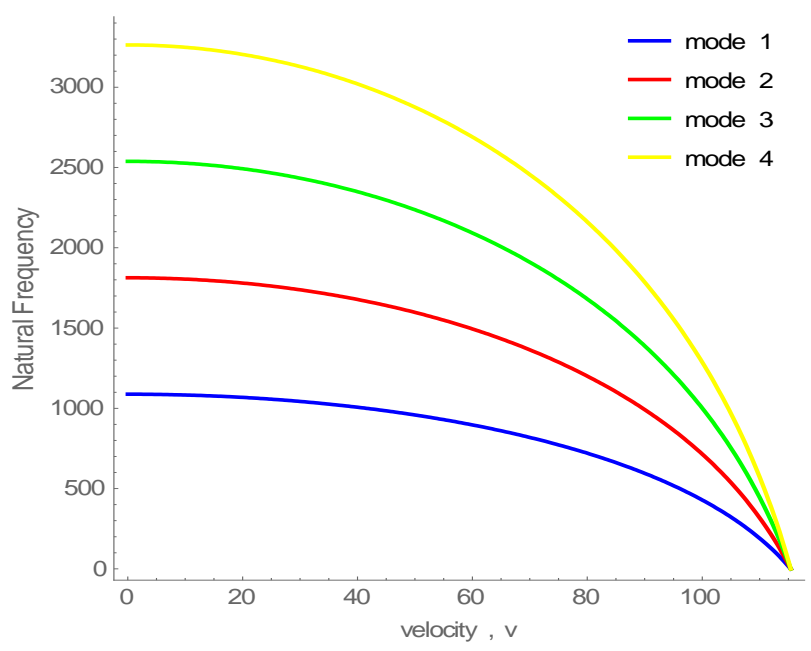

Figure 1c: Longitudinal Natural frequency 
Apparently, the critical velocities of pipes modeled with large strain deformation theory are higher than their outcomes in pipes modelled with small strain deformation theory. This realization is pronounced at elevated temperatures.

Meanwhile, longitudinal vibration of pipes with small strain deformation is independent of temperature. However, longitudinal critical velocity of pipes with large strain deformation reduces with increasing temperature.

\subsection{Effects of Pressure on Transverse and Axial Vibration:}

The effects of pressure on large strain deformation model as compared with small strain deformation model for transverse and longitudinal vibrations are shown in Figures $3 \mathrm{a}$ and 3b. Results were obtained for a pipe conveying two phase flows with a void fraction of 0.3 and mixture quality of 0.00037088 .

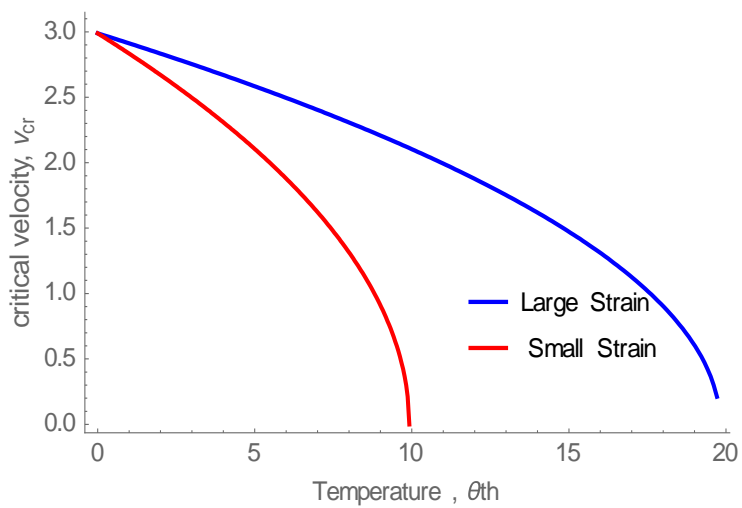

Figure 2a: Transverse critical velocities Vs temperature at void fraction $=0.3$, mixture quality $=0.00037088 ; \mathrm{Te}=0.1 ; \operatorname{Pr}=0.05$;

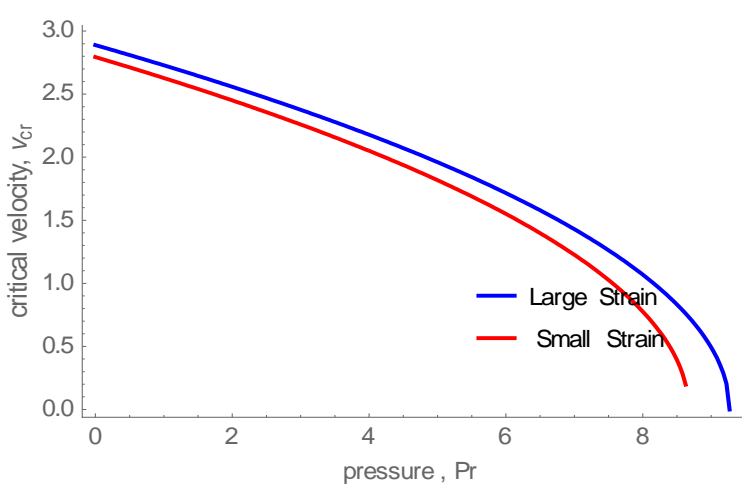

Figure 3a: Transverse critical velocities vs pressure at void fraction $=0.3$, mixture quality $=$ $0.00037088 ;$ th $=1.2 ; \mathrm{Te}=0.1$;
Clearly, the critical buckling velocity of the pipe persistently reduces with increasing pressure.

Longitudinal vibration of pipe modeled with small strain deformation theory does not respond to changes in pressure. On the other hand, the longitudinal critical velocity of pipe modeled with large strain deformation approach reduces with increasing pressure.

\subsection{Effects of Tension Transverse and Axial Vibration}

In contrast to small strain deformation models, the response of transverse and longitudinal vibrations to the effects of tension on pipe modeled with large strain deformation theory are shown in Figures $4 a$ and $4 \mathrm{~b}$. Results were obtained for pipe conveying two phase flows with void fraction of 0.3 and mixture quality of 0.00037088 . Clearly, the critical velocities of the pipe decreases with increasing tension.

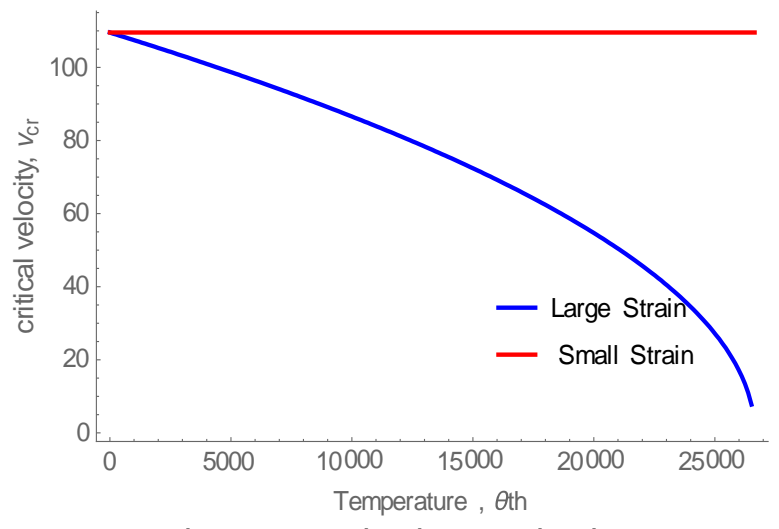

Figure 2b: Longitudinal critical velocities Vs temperature at void fraction $=0.3$, mixture quality $=0.00037088 ; \mathrm{Te}=0.1 ; \mathrm{Pr}=0.05$

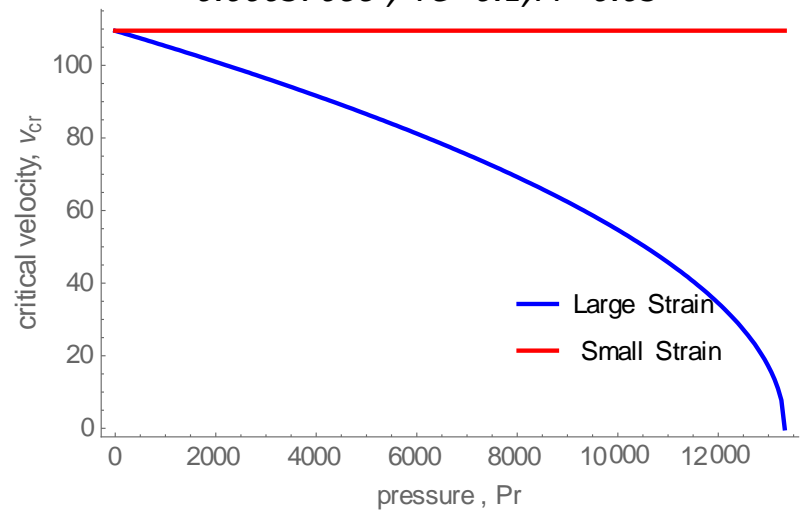

Figure 3b: Longitudinal critical velocities vs pressure at void fraction $=0.3$, mixture quality $=$ $0.00037088 ; \theta$ th $=1.2 ; \mathrm{Te}=0.1$ 


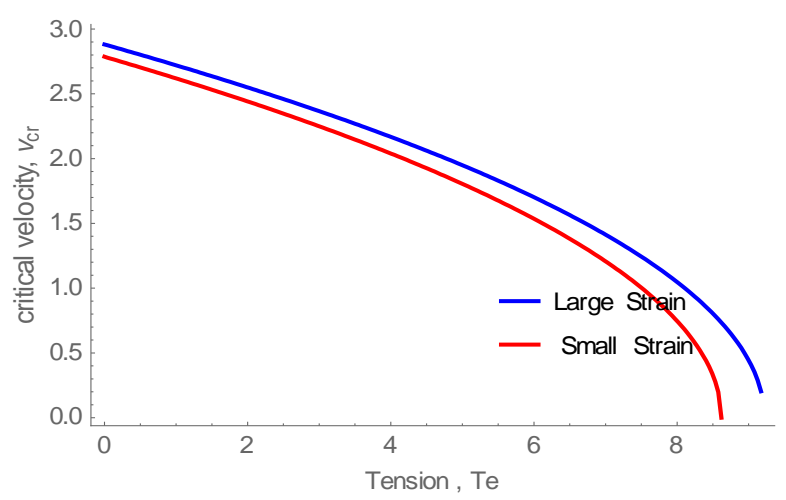

Figure 4a: Transverse critical velocities vs tension at void fraction $=0.3$, mixture quality $=$ $0.00037088 ;$ t $\theta$ th=1.2; $\mathrm{Pr}=0.05$;

\section{CONCLUSION AND RECOMMENDATIONS:}

In summary, this study examined the free vibration of a simply supported pipe conveying pressurized two phase flow modeled using the elastic large strain deformation theory. Nonlinear equations of motion and boundary conditions were obtained using Hamilton's principle. Approximate solutions of the dynamic system were obtained using the method multiple scale perturbation technique and natural frequencies of the dynamic system were derived from solutions of the leading order equation.

Clearly, buckling and flutter instabilities characterize large strain deformation model of flexible pipe conveying hot pressurized two phase fluids. This is consistent with the known response of small strain deformation models. However, in large strain model, the buckling velocity is sensitive to changes in temperature, pressure and tension. For pipes modeled with large strain model, it is evident that the critical velocity of the transverse vibration are higher compared with when small strain deformation theory is used.

To investigate the post buckling behavior of pipe with large strain deformation conveying two-phase fluid, it is recommended that this linear analysis dynamic behaviour pipe with large strain deformation conveying two-phase fluid extended as a basis for the nonlinear analysis.

\section{REFERENCES}

[1] S. Chucheepsakul, H. Tseng and T. Monprapussorn, "Large strain formulations of extensible flexible marine pipes transporting

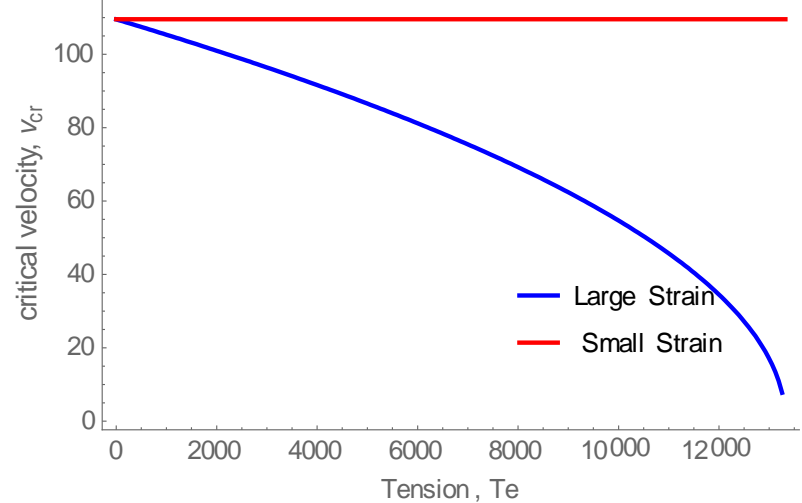

Figure 4b: Longitudinal critical velocities vs tension at void fraction $=0.3$, mixture quality $=$ $0.00037088 ; \theta$ th $=1.2 ; \mathrm{Pr}=0.05$;

fluid," Journal of Fluids and Structures, vol. 17, no. 2, pp. 185-224, 2003.

[2] M. Paidoussis, Fluid Structure Interactions : Slender Structures and Axial Flow., vol. 1, San Diego, CA: Academic Press Inc., 1998.

[3] S. Lee and J. Chung, "A new non-linear modelling for vibration analysis of a straight pipe conveying fluid," Journal of sound and vibration, vol. 254, no. 2, pp. 313-325, 2002.

[4] C. Semler, G. Li and M. Paidoussis, "The Nonlinear Equations of Motion of Pipes Conveying Fluid," Journal of Sound and Vibration, vol. 169, no. 5, pp. 577-599, 1994.

[5] M. Woldesemayat and A. Ghajar, "Comparison of void fraction correlations for different flow patterns in horizontal and upward inclined pipes," International Journal of Multiphase Flow, vol. 33, no. 4, pp. 347-370, 2007.

[6] M. Paidoussis and N. Issid, "Dynamic Stability of Pipes Conveying Fluid," Journal of Sound and Vibration, vol. 33, no. 3, pp. 267-294, 1974.

[7] A. Adegoke and A. Oyediran, "Natural frequencies, modes and critical velocities of Top Tensioned Cantilever Pipes Conveying Pressurized Steady Two-Phase Flow under Thermal Loading," Research on Engineering Structures \& Materials, vol. 4, no. 4, pp. 297323, 2018.

[8] Q. Qian, L. Wang and Q. Ni, "Instability of simply supported pipes conveying fluid under thermal loads," Mechanics Research Communications, vol. 36, no. 3, pp. 413-417, 2009. 